\title{
A device for improving the immunity of AC contactors during voltage dips
}

\author{
P. Andrada, J.I. Perat, G. Navarro \\ Departament d'Enginyeria Elèctrica \\ EPS d’Enginyeria de Vilanova i la Geltrú \\ Universitat Politècnica de Catalunya (UPC) \\ Grup d'Accionaments Elèctrics amb Commutació Electrònica (GAECE) \\ Av. Víctor Balaguer s/n, 08800 Vilanova i la Geltrú \\ Tel: +34 (93) 8967732, Fax: +34 (93) 8967700 \\ andrada@ee.upc.edu
}

\begin{abstract}
This paper describes an electronic device that enables AC contactors to ride through power quality disturbances. The proposed device is connected to the contactor coil and consists of a power conversion excitation and hold-in circuit, a control circuit, an immunity circuit and a shutdown circuit. It does not disturb contactor operation, is easy to use and can be built from cheap, commercially available components. Experimental tests have demonstrated the effectiveness of the proposed electronic device for improving the immunity of AC contactors during power quality disturbances, particularly voltage dips.
\end{abstract}

\section{Key words}

AC contactor, voltage dips, power quality, immunity.

\section{Introduction}

Power quality disturbances create major problems in continuous process industries. Momentary interruptions, voltage dips and dropouts are some of the most serious power quality problems faced by industrial customers. Power grid disturbances, which sometimes last less than a fraction of second, can cause a facility to shut down and interrupt production for considerable periods of time, which leads to loss of production and high costs [1-2]. AC contactors are often used to provide a remote switch between electric motors and the power grid that supplies them. Although electric motors can ride through some disturbances, AC contactors are extremely sensitive to this type of problem; several devices that are able to mitigate such disturbances have therefore been proposed. Most of these devices provide a momentary ride-through capability by maintaining the AC contactor in the closed position, by means of various procedures, so that the electric motor remains connected to the grid. The voltage regulator for contactor ride-through proposed by Kelley et al. is a good example of such a device [3].

In this paper we present an electronic device for improving the immunity of AC contactors during power quality disturbances, particularly voltage dips [4]. The electronic device shown in Figure 1 is connected to the contactor coil and consists of several circuits. Each of these circuits has a defined task and is supplied at a set voltage level. Some of them, such as the power conversion, excitation and hold-in circuit and the shutdown circuit, must safeguard the normal operation of the AC. The immunity circuit supplies the contactor coil when a disturbance occurs and is able to maintain contactor operation for a certain period of time, often for the entire duration of the disturbance. Finally, the control circuit decides which circuit must be activated at each moment. The paper is organized as follows. In Section II, following a short review of AC contactors, we describe the proposed electronic device and explain how it works. In Section III, we validate the effectiveness of the electronic device through a series of experimental tests. In Section IV, we present the conclusions.

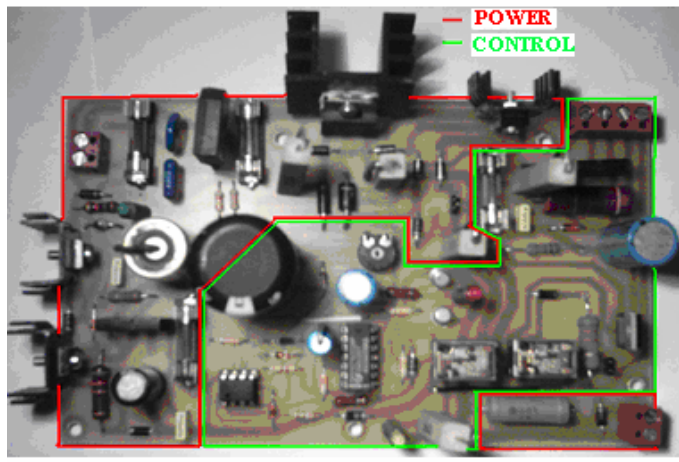

Fig. 1. Photograph of the proposed electronic device for AC contactor ride-through 


\section{Device description}

A contactor is an electrically controlled switch that is activated using a solenoid. The solenoid consists of an electromagnet that attracts a moveable, spring-loaded bar that is attached to electrical contacts. The contactor is operated by applying a voltage to the electromagnetic coil, which generates a current that induces a magnetic field to close the electrical contacts. AC contactors require a high current to close the contacts initially and a relatively low current to hold them closed during normal operation. Once the voltage is removed from the coil, the spring causes the contacts to open. Therefore, in AC contactors three different operational states may be distinguished: the excitation state, in which a high current is required to overcome the spring force; the hold-in state, in which a low current is all that is required to keep the contacts closed; and the shutdown state, in which the voltage in the contactor coil is removed by voluntary action and as a result the contacts open.

AC contactors provide a safe, easy and cheap way to control electric loads. They are widely used as remotely controlled switches between the power supply and motors that drive industrial processes (Fig. 2). AC contactors are more vulnerable to power quality disturbances than the motors they control. Often, the AC contactor is forced to open when a short voltage dip occurs and as a result the motor stops, whereas the motor alone, due to its inertia, could withstand this voltage dip.

In order to overcome this problem we present an electronic device for improving the immunity of AC contactors during power quality disturbances. In addition, the device does not disturb the normal contactor operation and is assembled from cheap, commercially available components. The proposed device adds a new operational state to the AC contactor, the immunity state, in which the contactor coil is supplied by an energy storage device when a power quality disturbance occurs in the AC voltage source. One of the most relevant features of the proposed electronic device together with an AC contactor is that every one of its operational states is associated with a circuit and every circuit is powered at a different voltage level through linear voltage regulators. The device consists of a power conversion, excitation and hold-in circuit, an immunity circuit, a control circuit and a shutdown circuit. Figure 3 shows a block diagram of the electronic device, in which the various circuits can be distinguished.

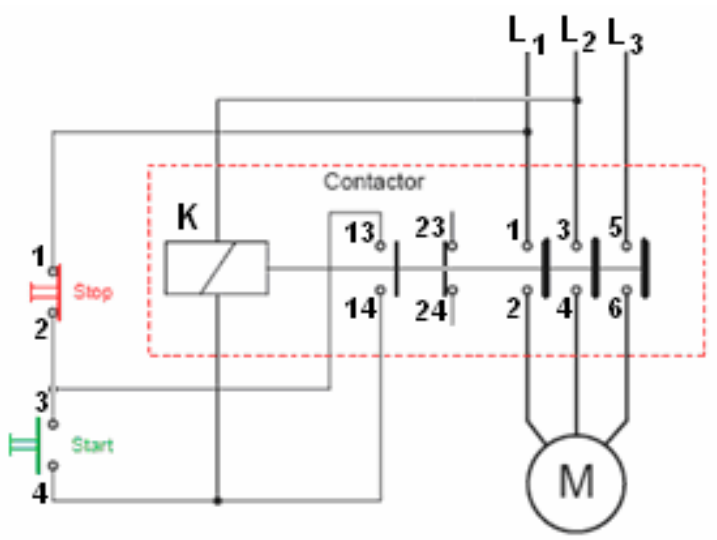

Fig. 2. Typical AC contactor connection diagram using start/stop pushbuttons

The power conversion, excitation and hold-in circuit includes a rectifier that is used to obtain a DC input voltage from the AC supply voltage and five linear voltage regulators that provide various DC voltage levels $(120 \mathrm{~V}, 56 \mathrm{~V}, 24 \mathrm{~V}, 12 \mathrm{~V}$ and $11 \mathrm{~V})$. Although a contactor is designed to operate on an AC voltage source, it can work equally well on a low DC voltage, as stated in [3]. Each of these voltages is appropriate for a specific contactor state, i.e., $120 \mathrm{~V}$ and $56 \mathrm{~V}$ are suitable for closing the contacts by overcoming the spring tension, 24 $\mathrm{V}$ is the voltage that produces the current needed to keep the contacts closed, $12 \mathrm{~V}$ is the voltage required by some ICs in the control circuit and $11 \mathrm{~V}$ is the voltage required by the shutdown circuit. The control circuit automatically provides the correct voltage according to the state of the contactor at any given time. The immunity circuit includes an energy storage device, that is, a capacitor that is always loaded. When a disturbance occurs in the AC voltage source the immunity circuit is activated by the control circuit and supplies the contactor coil. It is able to maintain the contacts closed for a period of time that depends on the time constant of the first order circuit, which is composed of the storage capacitor and the resistance of the electromagnetic coil. The shutdown circuit ensures that the contactor can be interrupted without delay when this is required by a voluntary action.

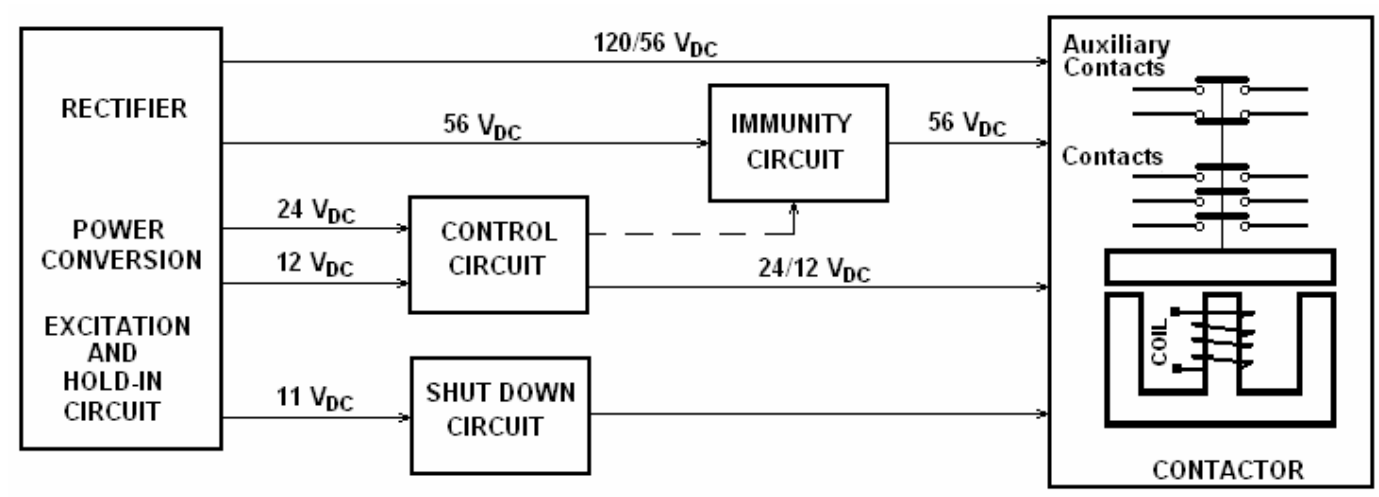

Fig. 3. General block diagram of the proposed electronic device 


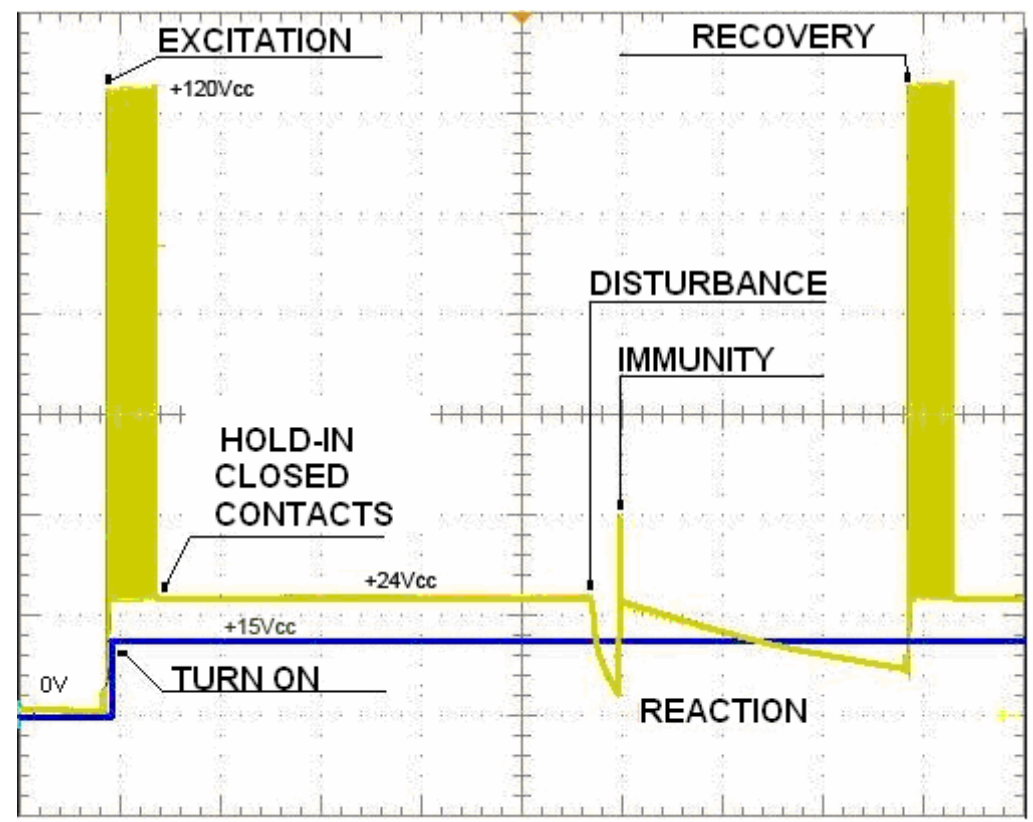

Fig. 4. Operational behavior of the electronic device

Figure 4 illustrates the behavior and associated voltages of the circuits, with the exception of the shutdown circuit. This figure shows the operational states-including the response to disturbances - of a contactor with the proposed electronic device, where the yellow waveform is the voltage in the contactor coil and the blue waveform is the voltage at the main contacts (15 V DC) - a test signal that indicates whether the contacts are open or closed.

\section{Experimental results}

The electronic device was tested in a setup in which a DC machine acting as a load was driven by a single-phase induction motor connected to a voltage dip generator through an AC contactor (Fig. 5). The response of several variables (motor current, AC supply voltage, speed and contactor coil voltage) to a short voltage dip when the proposed electronic device was not used is shown in Figure. 6 . The responses of the same variables to a short voltage dip when the AC contactor was connected to the power supply via the proposed electronic device can be seen in Figure 7. In the first case, the contacts open, the motor stops and the AC contactor must be restarted. In the second case, the motor is kept running and only a slight disturbance in its speed is noted. A transient torque is also observed but is not shown in Figure 7.

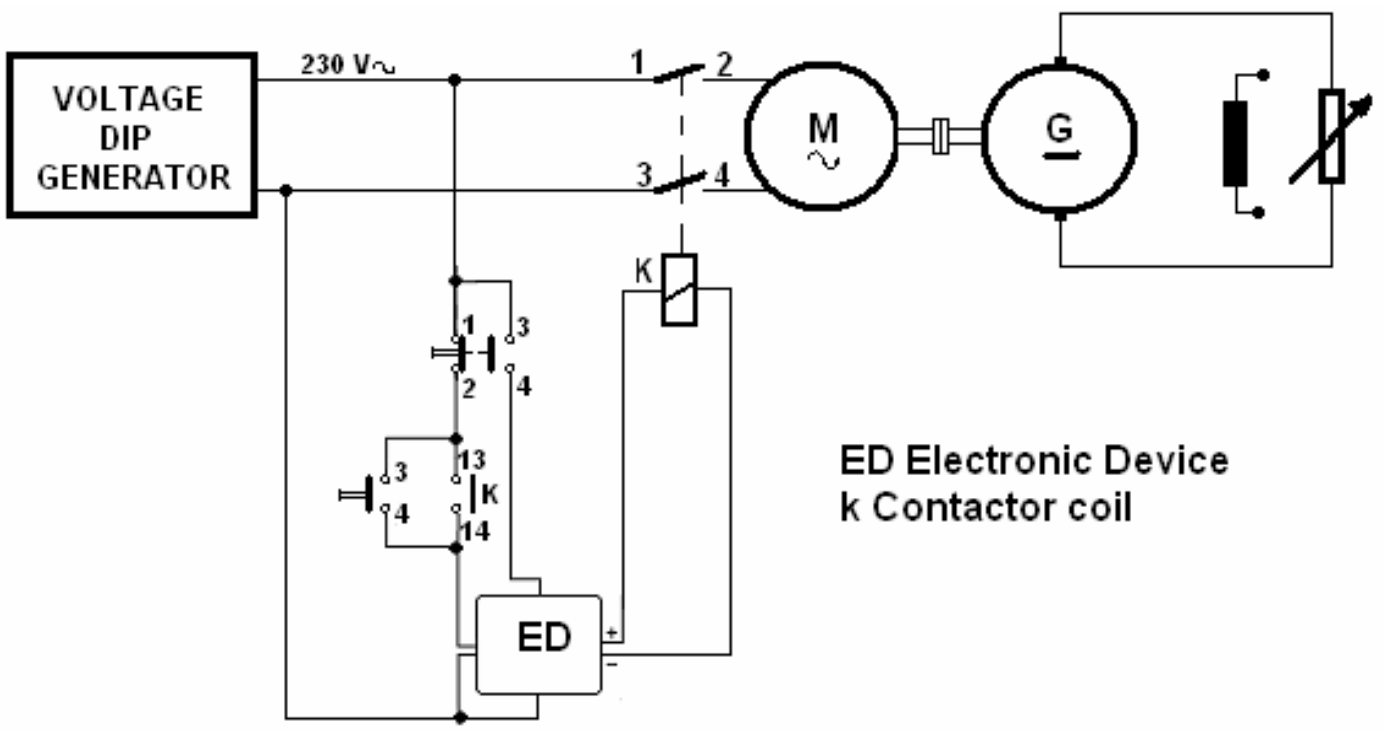

Fig. 5. Test setup, showing the proposed electronic device connection 


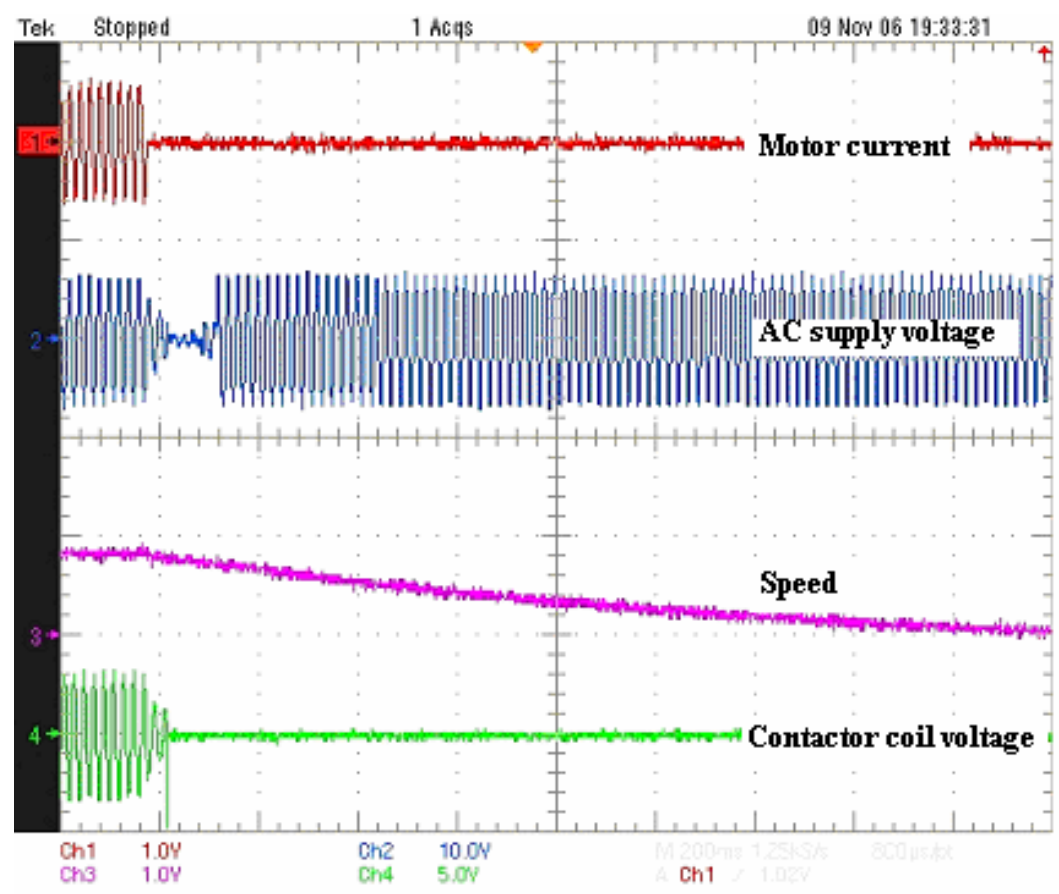

Fig. 6. Operational waveforms of an AC contactor, without the proposed electronic device, motor and AC voltage source, during a short voltage dip

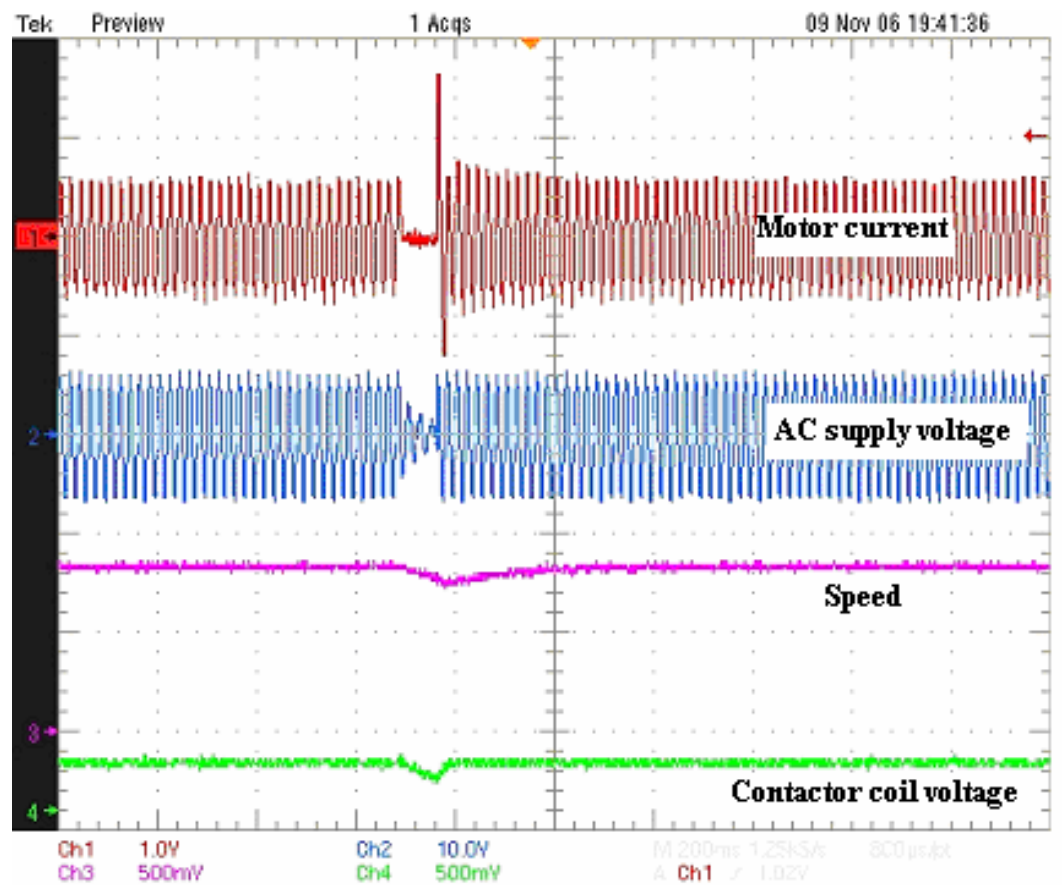

Fig. 7. Operation waveforms of an AC contactor, using the proposed electronic device, motor and AC voltage source, during a short voltage dip

The waveforms in Figure 8 correspond to the same conditions as in Figure 5, except that in this case the voltage dip lasts longer. In these circumstances, the voltage dip may cause the motor to stop. Nevertheless, it can clearly be observed that the action of the immunity circuit allows the main contacts to remain closed. The motor keeps running but there is a significant drop in its speed. By using the proposed device the AC contactor improves its immunity to power quality disturbances such as voltage dips and voltage drops, which prevents loss of production in industrial facilities. However, recent studies have pointed out that voltage dips and dip recovery devices may damage electromechanical equipment [5]. Consequently, before implementing the proposed device in an industrial process it is important to determine the balance between the savings it is expected to provide and the cost of replacing the motor that drives the process. 


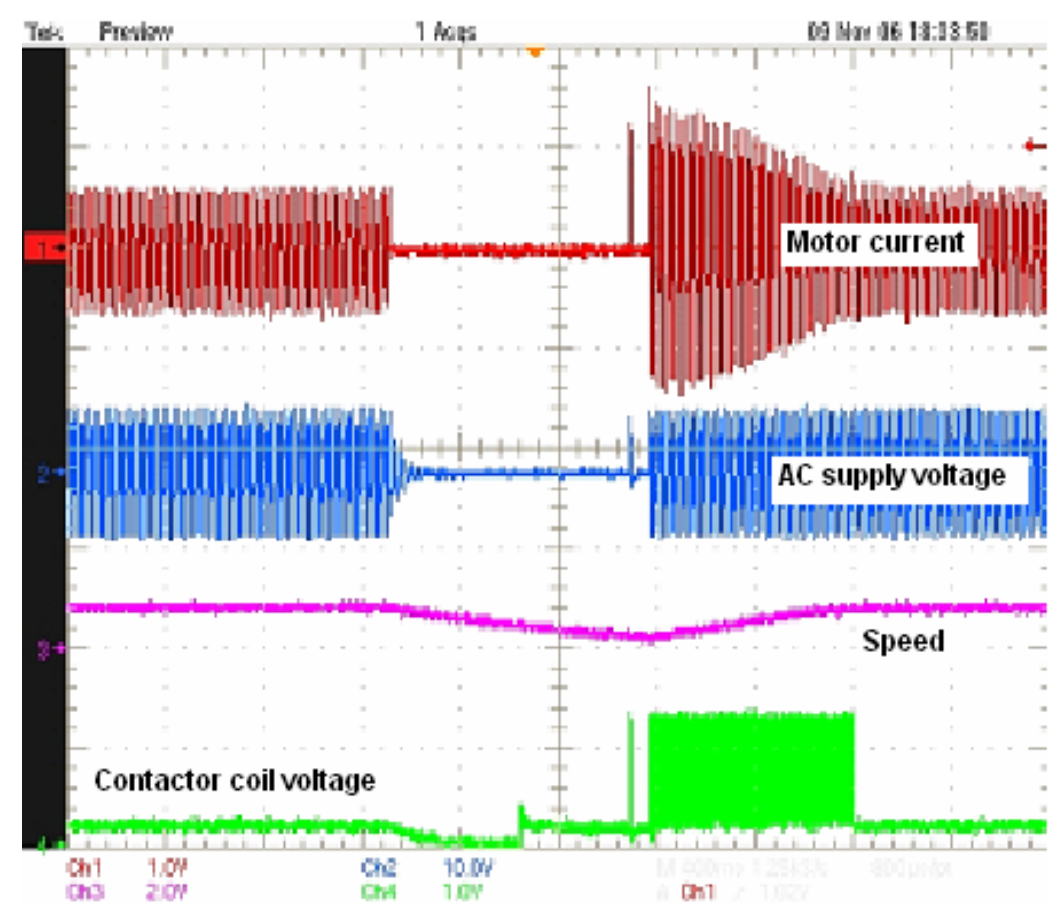

Fig. 8. Operational waveforms of an AC contactor, using the proposed electronic device, motor and AC voltage source, during a long voltage dip

\section{Conclusion}

In this paper we present an electronic device that allows an AC contactor to ride through power quality disturbances, in particular voltage dips. The proposed electronic device consists of several circuits and is connected to the contactor coil. The electronic device does not disturb the contactor operation, can be adapted to almost all types of electrical contactor, is easy to use and can be built from cheap, commercially available components. Experimental tests have demonstrated its usefulness in improving the immunity of the AC contactor during power voltage disturbances, particularly voltage dips.

\section{Acknowledgement}

This research was supported by the Spanish Ministry of Education and Science and the ERDF (DPI2006-09880).

\section{References}

[1] C.J. Melhorn, T.D. Davis, G.E. Beam. "Voltage sags: their impact on the utility and industrial customers". IEEE Transactions on Industry Applications, Vol. 34, No. 3, May/June 1988, pp. 549-558.

[2] M.F. McGranaghan, D.R. Mueller, M.J. Samotyj. "Voltage sags in industrial systems". IEEE Transactions on Industry Applications, Vol. 29, No. 2, March/April 1993, pp 397404.

[3] A. Kelley, J. Cavaroc, J. Ledford, L. Vassalli. "Voltage regulator for contactor ridethrough". IEEE Transactions on Industry Applications, Vol. 36, No. 2, March/April 2000 , pp. 697-703.

[4] P. Andrada, G. Navarro, J.I. Perat. "A new power supply system for AC contactor ride-through”. EPQU'07, October 2007, Barcelona.

[5] A. Bendre, D. Divan, W. Kranz, W.E. Brumsickle. "Are voltage sags destroying equipment?" IEEE Industry Magazine, Vol. 12, No. 4, July/August 2007, pp.12-21. 